\title{
Eigenvalues for anisotropic $p$-Laplacian under a Steklov-like boundary condition
}

\author{
Luminiţa Barbu
}

Dedicated to Professor Gheorghe Moroşanu on the occasion of his 70th anniversary.

Abstract. The eigenvalue problem

$$
-\operatorname{div}\left(\frac{1}{p} \nabla_{\xi}\left(F^{p}(\nabla u)\right)=\lambda a(x)|u|^{q-2} u,\right.
$$

with $q \in(1, \infty), p \in\left(\frac{N q}{N+q-1}, \infty\right), p \neq q$, subject to Steklov-like boundary condition,

$$
F^{p-1}(\nabla u) \nabla_{\xi} F(\nabla u) \cdot \nu=\lambda b(x)|u|^{q-2} u
$$

is investigated on a bounded Lipschitz domain $\Omega \subset \mathbb{R}^{N}, N \geq 2$. Here, $F$ stands for a $C^{2}\left(\mathbb{R}^{N} \backslash\{0\}\right)$ norm and $a \in L^{\infty}(\Omega), b \in L^{\infty}(\partial \Omega)$ are given nonnegative functions satisfying

$$
\int_{\Omega} a d x+\int_{\partial \Omega} b d \sigma>0
$$

Using appropriate variational methods, we are able to prove that the set of eigenvalues of this problem is the interval $[0, \infty)$.

Mathematics Subject Classification (2010): 35J60, 35J92, 35P30.

Keywords: Eigenvalues, anisotropic $p$-Laplacian, Steklov-like boundary condition, Sobolev spaces, variational methods.

\section{Introduction}

Let $F$ be a norm in $\mathbb{R}^{N}$, that is a nonnegative, positively homogeneous of degree 1 , convex function defined in $\mathbb{R}^{N}$. Moreover, we assume that $F \in C^{2}\left(\mathbb{R}^{N} \backslash\{0\}\right)$.

Next, let us introduce the so-called anisotropic $p$-Laplacian, defined as follows

$$
\mathcal{Q}_{p} u:=\operatorname{div}\left(\frac{1}{p} \nabla_{\xi}\left(F^{p}(\nabla u)\right) .\right.
$$


When $p=2, \mathcal{Q}_{2}$ is the anisotropic operator, also known as the Finsler-Laplace operator [6]. We point out that a typical example of $F$ satisfying the above conditions is the $l_{r}-$ norm

$$
F(\xi):=\left(\sum_{i=1}^{N}\left|\xi_{i}\right|^{r}\right)^{1 / r}, r>1,
$$

for which the operator $\mathcal{Q}_{p}$ has the form

$$
\Delta_{r, p} u:=\operatorname{div}\left(\|\nabla u\|_{r}^{p-r} \nabla^{r} u\right),
$$

where

$$
\nabla^{r} u:=\left(\left|\frac{\partial u}{\partial x_{1}}\right|^{r-2} \frac{\partial u}{\partial x_{1}}, \cdots,\left|\frac{\partial u}{\partial x_{N}}\right|^{r-2} \frac{\partial u}{\partial x_{N}}\right) .
$$

Note that $\Delta_{r, p}$ is a nonlinear operator unless $p=r=2$ when it reduces to the usual Laplacian operator. Two important special cases are $r=2$ and $p \in(1, \infty)$ when $\Delta_{2, p}$ coincides with the usual p-Laplace operator (see [12]) and the case $r=p$, when $\Delta_{p, p}$ is the so-called pseudo p-Laplacian. A physical motivation to study differential equations involving such operators is given by the fact that they appear in well-established models of surface energies in metallurgy, crystallography, crystalline fracture theory, or noise-removal procedures in digital image processing (see for instance, [9], [15], and references therein). Meanwhile, a geometric motivation for the investigation of such operators comes from the fact that such anisotropies appears naturally in the Finsler geometry, such as, for instance, the Minkowski geometry (see the seminal works of P. Finsler [7] and H. Minkowski [13]).

The paper concerns the study of the following Steklov-like eigenvalue problem for $\mathcal{Q}_{p}$ :

$$
\left\{\begin{array}{l}
-\mathcal{Q}_{p} u:=-\operatorname{div}\left(\frac{1}{p} \nabla_{\xi}\left(F^{p}(\nabla u)\right)=\lambda a(x)|u|^{q-2} u \text { in } \Omega,\right. \\
F^{p-1}(\nabla u) \nabla_{\xi} F(\nabla u) \cdot \nu=\lambda b(x)|u|^{q-2} u \text { on } \partial \Omega,
\end{array}\right.
$$

under the following hypotheses $\partial \Omega$

$$
\left(H_{p q}\right) q \in(1, \infty), p \in\left(\frac{N q}{N+q-1}, \infty\right), p \neq q ;
$$

$\left(H_{\Omega}\right) \Omega \subset \mathbb{R}^{N}, N \geq 2$, is a bounded domain with Lipschitz continuous boundary

$\left(H_{a b}\right) a, b \in L^{\infty}(\Omega)$ are given nonnegative functions satisfying

$$
\int_{\Omega} a d x+\int_{\partial \Omega} b d \sigma>0
$$

In $(1.1)_{2}, \nu$ stands for the outward unit normal to $\partial \Omega$.

The solution $u$ of (1.1) is understood in a weak sense, as an element of the Sobolev space $W^{1, p}(\Omega)$ satisfying equation $(1.1)_{1}$ in the sense of distributions and boundary condition $(1.1)_{2}$ in the sense of traces: 
Definition 1.1. $\lambda \in \mathbb{R}$ is an eigenvalue of problem (1.1) if there exists $u_{\lambda} \in W^{1, p} \backslash\{0\}$ such that for all $w \in W^{1, p}(\Omega)$

$$
\begin{aligned}
& \int_{\Omega}\left(F\left(\nabla u_{\lambda}\right)\right)^{p-1} \nabla_{\xi} F\left(\nabla u_{\lambda}\right) \cdot \nabla w d x \\
= & \lambda\left(\int_{\Omega} a\left|u_{\lambda}\right|^{q-2} u_{\lambda} w d x+\int_{\partial \Omega} b\left|u_{\lambda}\right|^{q-2} u_{\lambda} w d \sigma\right) .
\end{aligned}
$$

Indeed, according to a Green type formula (see [4], p. 71), $u \in W^{1, p}(\Omega)$ is a solution of (1.1) if and only if it satisfies (1.3).

Our goal is to determine the set of all eigenvalues of problem (1.1). Fortunately we are able to offer a complete description of this set.

The main result of our paper is given by the following theorem

Theorem 1.2. Assume that $\left(H_{p q}\right),\left(H_{\Omega}\right)$ and $\left(H_{a b}\right)$ above are fulfilled. Then the set of eigenvalues of problem $(1.1)$ is $[0, \infty)$.

It is worth pointing out that this nice result is due to the fact that operator $\mathcal{Q}_{p}$ is nonhomogeneous $(p \neq q)$. The homogeneous case $(p=q)$ is more delicate. For example, if $p=q$ and either $a \equiv 1, b \equiv 0$ or $a \equiv 0, b \equiv 1$ and $F$ is the usual euclidian norm, then the eigenvalue set of the corresponding (Neumann type) problem is fully known only if $p=q=2$; otherwise, i.e. if $p=q \in(1, \infty) \backslash\{2\}$, then it is only known that, as a consequence of the Ljusternik-Schnirelman theory, there exists a sequence of positive eigenvalues of problem (1.1) with $Q=-\Delta_{p}$ (see, e.g., [11]), but this sequence may not constitute the whole eigenvalue set.

Regarding the assumption $p \in\left(\frac{N q}{N+q-1}, \infty\right)$ we point out that this is directly related to the well-known embeddings $W^{1, p}(\Omega) \hookrightarrow L^{q}(\Omega)$ which hold in the cases: (1) $1 \leq q \leq p^{*}=p N /(N-p)$, if $1<p<N$; (2) $p \leq q<\infty$, if $p=N$; (3) $q=\infty$, if $p>N$. Moreover, these embeddings are compact when $1 \leq q<p^{*}$ in case (1), all $q$ in case (2), and when reinterpreted as $W^{1, p}(\Omega) \hookrightarrow C^{1}(\bar{\Omega})$ in case (3). We also have trace compactly embeddings $W^{1, p}(\Omega) \hookrightarrow L^{q}(\partial \Omega)$ for all $1 \leq p \leq q<p(N-1) /(N-p)$ if $1 \leq p<N$, and similarly as before in the other ranges of $p$ (see [1], [3, Section 9.3]).

Also, we restrict ourselves to functions $a \in L^{\infty}(\Omega), b \in L^{\infty}(\partial \Omega)$ since assuming weaker regularity for these functions leads to similar results without essential changes.

The Dirichlet eigenvalue problem associated with operator $-\mathcal{Q}_{p}$ for $q=2$ has been studied in [5]. As far as the problem (1.1) is concerned, a separate analysis is needed since some specific situations have to be addressed, including those related to the trace on $\partial \Omega$ and the fact that the eigenfunctions of our problem belong to the set $\mathcal{C}$ (see Section 2, (2.2) for the definition of $\mathcal{C}$ ). It is worth pointing out that results concerning the existence and nonexistence of solutions for the case of $p$-Laplacian under Dirichlet boundary conditions and appropriate assumptions on $\Omega$ have been obtained by M. Ôtani in the well known paper [14].

\section{Preliminary results}

Our hypotheses $\left(H_{p q}\right),\left(H_{\Omega}\right),\left(H_{a b}\right)$ will be assumed throughout this paper. Testing equation (1.3) against $w=u_{\lambda}$ we observe that the eigenvalues of problem (1.1) 
cannot be negative numbers. It is also obvious that $\lambda_{0}=0$ is an eigenvalue of this problem and the corresponding eigenfunctions are the nonzero constant functions. So any other eigenvalue belongs to $(0, \infty)$.

If we assume that $\lambda>0$ is an eigenvalue of problem (1.1) and choose $w \equiv 1$ in (1.3) we deduce that every eigenfunction $u_{\lambda}$ corresponding to $\lambda$ satisfies the equation

$$
\int_{\Omega} a\left|u_{\lambda}\right|^{q-2} u_{\lambda} d x+\int_{\partial \Omega} b\left|u_{\lambda}\right|^{q-2} u_{\lambda} d \sigma=0 .
$$

So all eigenfunctions corresponding to positive eigenvalues necessarily belong to the set

$$
\mathcal{C}:=\left\{u \in W^{1, p}(\Omega) ; \int_{\Omega} a|u|^{q-2} u d x+\int_{\partial \Omega} b|u|^{q-2} u d \sigma=0\right\} .
$$

This is a symmetric cone and we can see that $\mathcal{C}$ is a weakly closed subset of $W^{1, p}(\Omega)$. Indeed, let $\left(u_{n}\right)_{n} \subset \mathcal{C}$ such that $u_{n} \rightarrow u_{0}$ in $W^{1, p}(\Omega)$. From assumption $\left(H_{p q}\right)$, $W^{1, p}(\Omega) \hookrightarrow L^{q}(\Omega)$ and $W^{1, p}(\Omega) \hookrightarrow L^{q}(\partial \Omega)$ compactly, hence there exists a subsequence of $\left(u_{n}\right)_{n}$, which is also denoted $\left(u_{n}\right)_{n}$, such that

$$
u_{n} \rightarrow u_{0} \text { in } L^{q}(\Omega), u_{n} \rightarrow u_{0} \text { in } L^{q}(\partial \Omega) .
$$

By Lebesgue's Dominated Convergence Theorem (see also [3, Theorem 4.9]) we obtain $u_{0} \in \mathcal{C}$.

In addition, $\mathcal{C}$ has nonzero elements (see [2, Section 2]).

Now let us define the positively homogeneous of order $p$ functional

$$
J: W^{1, p}(\Omega) \rightarrow \mathbb{R}, J(w):=\int_{\Omega}(F(\nabla w))^{p} d x \forall w \in W .
$$

Standard arguments can be used in order to deduce that functional $J$ is convex and weakly lower semicontinuous (see, for instance [16, Proposition 25.20]).

Consider the minimization problem

$$
\mu:=\inf _{w \in \mathcal{C}_{1}} J(w),
$$

where

$$
\mathcal{C}_{1}:=\mathcal{C} \cap\left\{u \in W^{1, p}(\Omega) ; \int_{\Omega} a|u|^{q} d x+\int_{\partial \Omega} b|u|^{q} d \sigma=1\right\} .
$$

The next result states that $J$ attains its minimal value and this value is positive.

Lemma 2.1. For each $p>1$ there exists $u_{*} \in \mathcal{C}_{1}$ such that

$$
\mu:=J\left(u_{*}\right)=\inf _{w \in \mathcal{C}_{1}} J(w)>0 .
$$

Proof. Let $\left(u_{n}\right)_{n} \subset \mathcal{C}_{1}$ be a minimizing sequence for $J$, i. e.,

$$
J\left(u_{n}\right) \rightarrow \inf _{w \in \mathcal{C}_{1 q}} J(w):=\mu .
$$

We can prove that $\left(u_{n}\right)_{n}$ is bounded in $W^{1, p}(\Omega)$. Assume the contrary, that there exists a subsequence of $\left(u_{n}\right)_{n}$, again denoted $\left(u_{n}\right)_{n}$, such that $\left\|u_{n}\right\|_{W^{1, p}(\Omega)} \rightarrow \infty$ as $n \rightarrow \infty$. Define

$$
v_{n}=\frac{u_{n}}{\left\|u_{n}\right\|_{W^{1, p}(\Omega)}} \quad \forall n \in \mathbb{N} .
$$


Clearly sequence $\left(v_{n}\right)_{n}$ is bounded in $W^{1, p}(\Omega)$ so there exist a $v \in W^{1, p}(\Omega)$ and a subsequence of $\left(v_{n}\right)_{n}$, again denoted $\left(v_{n}\right)_{n}$, such that

$$
v_{n} \rightarrow v \text { in } W^{1, p}(\Omega) .
$$

Taking into account assumption $\left(H_{p q}\right)$ we obtain that $W^{1, p}(\Omega) \hookrightarrow L^{q}(\Omega)$ and $W^{1, p}(\Omega) \hookrightarrow L^{q}(\partial \Omega)$ compactly, therefore, up to a subsequence, we have

$$
v_{n} \rightarrow v \text { in } L^{q}(\Omega), v_{n} \rightarrow v \text { in } L^{q}(\partial \Omega) .
$$

As $\left\|v_{n}\right\|_{W^{1, p}(\Omega)}=1 \forall n \in \mathbb{N}$ we have $\|v\|_{W^{1, p}(\Omega)}=1$, and

$$
\begin{aligned}
\int_{\Omega}(F(\nabla v))^{p} d x & \leq \liminf _{n \rightarrow \infty} \int_{\Omega}\left(F\left(\nabla v_{n}\right)\right)^{p} d x \\
& =\liminf _{n \rightarrow \infty} \frac{1}{\left\|u_{n}\right\|_{W^{1, p}(\Omega)}^{p}} J\left(u_{n}\right)=0
\end{aligned}
$$

which shows that $v$ is a constant function. On the other hand, since $\left(v_{n}\right)_{n} \subset \mathcal{C}$ and $\mathcal{C}$ is weakly closed in $W^{1, p}(\Omega)$, we infer that $v \in \mathcal{C}$, hence $v \equiv 0$. But this contradicts the fact that $\|v\|_{W^{1, p}(\Omega)}=1$. Therefore, $\left(u_{n}\right)_{n}$ is indeed bounded in $W^{1, p}(\Omega)$, thus, by passing to a subsequence, we can assume that $\left(u_{n}\right)_{n}$ converges weakly to a function $u_{*} \in W^{1, p}(\Omega)$ and

$$
u_{n} \rightarrow u_{*} \text { in } L^{q}(\Omega), u_{n} \rightarrow u_{*} \text { in } L^{q}(\partial \Omega) .
$$

By Lebesgue's Dominated Convergence Theorem we obtain $u_{*} \in \mathcal{C}_{1}$, so the weak lower semicontinuity of $J$ leads to $\mu=J\left(u_{*}\right)$. In addition, $J\left(u_{*}\right)>0$. Indeed, assuming by contradiction that $J\left(u_{*}\right)=0$ would imply that $u_{*} \equiv$ Const., which is impossible because $u_{*} \in \mathcal{C}_{1}$.

\section{Proof of the main result}

The following lemma plays a crucial role in the proof of our main theorem

Lemma 3.1. Assume that $\left(H_{p q}\right),\left(H_{\Omega}\right)$ and $\left(H_{a b}\right)$ above are fulfilled. Let $u_{*} \in W^{1, p}(\Omega)$ be a minimizer of the functional $J$ defined by (2.3) on the set

$$
\mathcal{C}_{1}:=\mathcal{C} \cap\left\{u \in W^{1, p}(\Omega) ; \int_{\Omega} a|u|^{q} d x+\int_{\partial \Omega} b|u|^{q} d \sigma=1\right\} .
$$

Then $u_{*}$ is an eigenfunction of problem (1.1) with eigenvalue $\mu=\inf _{w \in \mathcal{C}_{1}} J(w)$.

Proof. Since the constraint $\mathcal{C}_{1}$ is no more a $C^{1}$ manifold if $q<2$, we can not use a reasoning based on Lagrange Multipliers Rule. In order to avoid this inconvenience let us define the functional

$$
\begin{aligned}
J_{\mu}: W^{1, p}(\Omega) & \rightarrow \mathbb{R}, J_{\mu}(u)=\int_{\Omega}(F(\nabla u))^{p} d x \\
& -\mu\left(\int_{\Omega} a|u|^{q} d x+\int_{\partial \Omega} b|u|^{q} d \sigma\right)^{\frac{p}{q}} \forall u \in W^{1, p}(\Omega) .
\end{aligned}
$$


Standard arguments can be used in order to deduce that $J_{\mu} \in C^{1}\left(W^{1, p}(\Omega) ; \mathbb{R}\right)$, with the derivative given by

$$
\begin{aligned}
\left\langle J_{\mu}^{\prime}(u), w\right\rangle & =p \int_{\Omega}\left(F(\nabla u)^{p-1} \nabla_{\xi} F(\nabla u) \cdot \nabla w d x\right. \\
& -\mu p\left(\int_{\Omega} a|u|^{q} d x+\int_{\partial \Omega} b|u|^{q} d \sigma\right)^{\frac{p}{q}-1} \\
& \cdot\left(\int_{\Omega} a|u|^{q-2} u w d x+\int_{\partial \Omega} b|u|^{q-2} u w d \sigma\right)
\end{aligned}
$$

for all $u, w \in W^{1, p}(\Omega)$.

It is obviously that $u_{*}$ is an eigenfunction of problem (1.1) with eigenvalue $\mu$ if and only if $u_{*}$ is a critical point of $J_{\mu}$, i. e. $J_{\mu}^{\prime}\left(u_{*}\right)=0$. In order to show this, we fix $v \in \operatorname{Lip}(\Omega)$ arbitrarily. For each $n \in \mathbb{N}^{*}$ define $f_{n}: \mathbb{R} \rightarrow \mathbb{R}$,

$$
f_{n}(s)=\int_{\Omega} a\left|u_{*}+\frac{1}{n} v+s\right|^{q} d x+\int_{\partial \Omega} b\left|u_{*}+\frac{1}{n} v+s\right|^{q} d \sigma \forall s \in \mathbb{R} .
$$

It is easily seen that $f_{n}$ is coercive, since we have

$$
\begin{aligned}
f_{n}(s) & \geq 2^{-q}|s|^{q}\left(\|a\|_{L^{\infty}(\Omega)}|\Omega|_{N}+\|b\|_{L^{\infty}(\partial \Omega)}|\partial \Omega|_{N-1}\right) \\
& -\int_{\Omega} a\left|u_{*}+\frac{1}{n} v\right|^{q} d x-\int_{\partial \Omega} b\left|u_{*}+\frac{1}{n} v\right|^{q} d \sigma,
\end{aligned}
$$

where $|\cdot|_{N}$ and $|\cdot|_{N-1}$ denote the Lebesgue measures of the two sets. We have also used the inequality

$$
|x|^{q} \leq(|x+y|+|y|)^{q} \leq 2^{q}\left(|x+y|^{q}+|y|^{q}\right) \forall x, y \in \mathbb{R}, q>1 .
$$

Moreover, function $f_{n}$ is continuous differentiable on $\mathbb{R}$ (see [8, Theorem 2.27]) and convex (its derivative is an increasing function). Therefore, for all $n \in \mathbb{N}^{*}, f_{n}$ admits a minimum point $s_{n}$, such that $f_{n}^{\prime}\left(s_{n}\right)=0$, that is

$$
\begin{aligned}
\int_{\Omega} a \mid u_{*} & +\frac{1}{n} v+\left.s_{n}\right|^{q-2}\left(u_{*}+\frac{1}{n} v+s_{n}\right) d x \\
& +\int_{\partial \Omega} b\left|u_{*}+\frac{1}{n} v+s_{n}\right|^{q-2}\left(u_{*}+\frac{1}{n} v+s_{n}\right) d \sigma=0 .
\end{aligned}
$$

We denote

$$
u_{n}:=u_{*}+1 / n v+s_{n} \forall n \in \mathbb{N}^{*} .
$$

From (3.4) we derive that $\left(u_{n}\right)_{n} \subset \mathcal{C}$.

Next, we claim that the sequence $\left(n s_{n}\right)_{n}$ is bounded. Arguing by contradiction, let us assume that, up to a sequence, $n s_{n} \rightarrow \infty$ or $n s_{n} \rightarrow-\infty$ as $n \rightarrow \infty$. Taking into account that $v \in \operatorname{Lip}(\Omega)$ there exists $N_{1}$ large enough such that we have either

$$
v(\cdot)+n s_{n}>0 \text { in } \Omega \text {, or } v(\cdot)+n s_{n}<0 \text { in } \Omega \forall n \geq N_{1} .
$$


Since the function $\gamma \rightarrow\left|u^{*}+\gamma\right|^{q-2}\left(u^{*}+\gamma\right)$ is strictly increasing on $\mathbb{R}$, we get

$$
\begin{aligned}
0 & =\int_{\Omega} a\left|u_{n}\right|^{q-2} u_{n} d x+\int_{\partial \Omega} b\left|u_{n}\right|^{q-2} u_{n} d \sigma \\
& >\int_{\Omega} a\left|u^{*}\right|^{q-2} u^{*} d x+\int_{\partial \Omega} b\left|u^{*}\right|^{q-2} u^{*} d \sigma=0 \forall n \geq N_{1},
\end{aligned}
$$

if $v(\cdot)+n s_{n}>0$ in $\Omega$, or the reverse inequality in the second situation, when

$$
v(\cdot)+n s_{n}<0 \text { in } \Omega .
$$

In both cases we get a contradiction.

We point out that inequality in relation (3.6) is strictly. Indeed, we note that (1.2) implies that either $|\{x \in \Omega ; a(x)>0\}|_{N}>0$ or $a=0$ a.e. in $\Omega$ and

$$
|\{x \in \partial \Omega ; b(x)>0\}|_{N-1}>0,
$$

hence we can not have equality between the two terms containing integrals.

Consequently, $\left(n s_{n}\right)_{n}$ should be bounded. This in turn implies there exists $S \in \mathbb{R}$ such that, up to a subsequence, $n s_{n} \rightarrow S$ as $n \rightarrow \infty$.

We note that the subsequence of $\left(u_{n}\right)_{n}$, denoted $\left(u_{n}\right)_{n}$ again, with the property that $\left(n s_{n}\right)_{n}$ has the limit $S$, converges in $W^{1, p}(\Omega)$, more exactly,

$$
u_{n} \rightarrow u_{*} \text { and } n\left(u_{n}-u_{*}\right) \rightarrow v+S \text { in } W^{1, p}(\Omega) \text { as } n \rightarrow \infty .
$$

We also note that from (3.7), combining with $u_{*} \not \equiv 0$, there exists $N_{2}$ large enough, such that $\left(u_{n}\right)_{n} \subset \mathcal{C} \backslash\{0\} \forall n \geq N_{2}$. Next, using this subsequence, we are going to construct a minimizing sequence for $J_{\mu}$ restricted to the constraint set $\mathcal{C}_{1}$. In this respect, we can define

$$
t_{n}:=\left(\left\|a^{1 / q} u_{n}\right\|_{L^{q}(\Omega)}^{q}+\left\|b^{1 / q} u_{n}\right\|_{L^{q}(\partial \Omega)}^{q}\right)^{1 / q}, z_{n}:=\frac{u_{n}}{t_{n}},
$$

for all $n$ sufficiently large. Obviously, we have

$$
\begin{aligned}
t_{n} \rightarrow & \int_{\Omega} a\left|u_{*}\right|^{q} d x+\int_{\partial \Omega} b\left|u_{*}\right|^{q} d \sigma=1, \\
& \quad\left(z_{n}\right)_{n} \subset \mathcal{C}_{1}, z_{n} \rightarrow u_{*} \text { in } W^{1, p}(\Omega) \text { as } n \rightarrow \infty .
\end{aligned}
$$

Next, we claim that sequence $\left(n\left(t_{n}-1\right)\right)_{n}$ is bounded. In order to proof this, we first show that $\left(n\left(t_{n}^{1 / q}-1\right)\right)_{n}$ is bounded. To this aim, we define the functional

$$
\mathcal{I}_{q}: W^{1, p}(\Omega) \rightarrow \mathbb{R}, \mathcal{I}_{q}(u):=\int_{\Omega} a|u|^{q} d x+\int_{\partial \Omega} b|u|^{q} d \sigma \forall u \in W^{1, p}(\Omega) .
$$

Under assumption $\left(H_{p q}\right)$, it is known that $\mathcal{I}_{q} \in C^{1}\left(W^{1, p}(\Omega) ; \mathbb{R}\right)$ (see, for instance [11]) and for all $u, w \in W^{1, p}(\Omega)$,

$$
\left\langle\mathcal{I}_{q}^{\prime}(u), w\right\rangle=q\left(\int_{\Omega} a|u|^{q-2} u w d x+\int_{\partial \Omega} b|u|^{q-2} u w d \sigma\right) .
$$

Since $\mathcal{I}_{q}\left(u_{*}\right)=1$, we note that for all $n \in \mathbb{N}^{*}$,

$$
n\left(t_{n}^{1 / q}-1\right)=\frac{\mathcal{I}_{q}\left(u_{n}\right)-\mathcal{I}_{q}\left(u_{*}\right)}{\frac{1}{n}} .
$$


Now, taking into account that $\mathcal{I}_{q}^{\prime} \in\left(W^{1, p}(\Omega)\right)^{*}$, we get

$$
\begin{aligned}
\lim _{n \rightarrow \infty} n\left(t_{n}^{1 / q}-1\right) & =\lim _{n \rightarrow \infty} n\left(\mathcal{I}_{q}\left(u_{n}\right)-\mathcal{I}_{q}\left(u_{*}\right)\right) \\
& =\lim _{n \rightarrow \infty}\left\langle\mathcal{I}_{q}^{\prime}\left(u_{*}\right), n\left(u_{n}-u_{*}\right)\right\rangle+o\left(n ; u_{*}, v\right) \\
& =\left\langle\mathcal{I}_{q}^{\prime}\left(u_{*}\right), v+S\right\rangle=\left\langle\mathcal{I}_{q}^{\prime}\left(u_{*}\right), v\right\rangle,
\end{aligned}
$$

where $o\left(n ; u_{*}, v\right)$ is a notation for the term which tends to zero in the definition of the Fréchet differential of $\mathcal{I}_{q}$ at $u_{*}$, that is $o\left(n, u_{*}, v\right) \rightarrow 0$ as $n \rightarrow \infty$. Therefore, there exists $K>0$ such that $n\left|t_{n}^{1 / q}-1\right| \leq K$, or equivalently

$$
0<1-\frac{K}{n} \leq t_{n}^{1 / q} \leq 1+\frac{K}{n}
$$

for all $n \in \mathbb{N}^{*}, n$ large enough, which implies

$$
n\left(\left(1-\frac{K}{n}\right)^{q}-1\right) \leq n\left(t_{n}-1\right) \leq n\left(\left(1+\frac{K}{n}\right)^{q}-1\right)
$$

for all $n$ sufficiently large. It is elementary to check that

$$
\lim _{x \rightarrow 0_{+}} \frac{(1+K x)^{q}-1}{x}=q K, \lim _{x \rightarrow 0_{+}} \frac{(1-K x)^{q}-1}{x}=-q K .
$$

This in combination with (3.13) implies that the sequence $\left(n\left(t_{n}-1\right)\right)_{n}$ is bounded, thus, by possibly passing to a subsequence, there exists $T \in \mathbb{R}$, such that $n\left(t_{n}-1\right) \rightarrow T$ as $n \rightarrow \infty$.

Now, it is easy to observe that $u_{*}$ minimizes functional $J_{\mu}$ over $\mathcal{C}_{1}$. By using the minimality of $u_{*}$ and the fact that $\left(z_{n}\right)_{n} \subset \mathcal{C}_{1}$ we obtain that

$$
0 \leq \lim _{n \rightarrow \infty} \frac{J_{\mu}\left(z_{n}\right)-J_{\mu}\left(u_{*}\right)}{\frac{1}{n}} .
$$

Since functional $J_{\mu} \in C^{1}\left(W^{1, p}(\Omega) ; \mathbb{R}\right)$, we have

$$
n\left(J_{\mu}\left(z_{n}\right)-J_{\mu}\left(u_{*}\right)\right)=\left(\left\langle J_{\mu}^{\prime}\left(u_{*}\right), n\left(z_{n}-u_{*}\right)\right\rangle+o\left(n ; u_{*}, v\right)\right.
$$

with $o\left(n ; u_{*}, v\right) \rightarrow 0$ as $n \rightarrow \infty$. Taking into account (3.5) and (3.8) we can see that

$$
n\left(z_{n}-u_{*}\right)=\frac{1}{t_{n}}\left(n u_{*}\left(1-t_{n}\right)+v+n s_{n}\right) \rightarrow-T u_{*}+v+S \text { as } n \rightarrow \infty .
$$

It follows from (3.14)-(3.16) that

$$
0 \leq\left\langle J_{\mu}^{\prime}\left(u_{*}\right), v+S-T u_{*}\right\rangle .
$$

From $(3.2)$, Lemma 2.1, and $u_{*} \in \mathcal{C}_{1}$, we get that $\left\langle J_{\mu}^{\prime}\left(u_{*}\right), u_{*}\right\rangle=0,\left\langle J_{\mu}^{\prime}\left(u_{*}\right), S\right\rangle=0$, hence (3.17) implies

$$
0 \leq\left\langle J_{\mu}^{\prime}\left(u_{*}\right), v\right\rangle
$$

A similar reasoning with $-v$ instead of $v$ shows that $0=\left\langle J_{\mu}^{\prime}\left(u_{*}\right), v\right\rangle$.

The conclusion then follows by exploiting the density of Lipschitz functions in $W^{1, p}(\Omega)$ which is true according to assumption $\left(H_{\Omega}\right)$ (see [10, Theorm 3.6]. 
Proof of Theorem 1.2. By Lemma 3.1, there exists an eigenfunction $u_{*}$ of problem (1.1) corresponding to eigenvalue $\mu=\inf _{w \in \mathcal{C}_{1}} J(w)>0$, thus

$$
\begin{gathered}
\int_{\Omega}\left(F\left(\nabla u_{*}\right)\right)^{p-1} \nabla_{\xi} F\left(\nabla u_{*}\right) \cdot \nabla w d x \\
=\mu\left(\int_{\Omega} a\left|u_{*}\right|^{q-2} u_{*} w d x+\int_{\partial \Omega} b\left|u_{*}\right|^{q-2} u_{*} w d \sigma\right)
\end{gathered}
$$

for all $w \in W^{1, p}(\Omega)$.

Consider $\lambda>0$ fixed. Let $\tau>0$. If we take $u_{*}$ of the form $u_{*}=\tau v_{*}$ in (3.18) and taking into account that $F$ and $\nabla_{\xi} F$ are positively homogeneous of degree 1 and 0 , respectively, we derive

$$
\begin{gathered}
\int_{\Omega}\left(F\left(\nabla v_{*}\right)\right)^{p-1} \nabla_{\xi} F\left(\nabla v_{*}\right) \cdot \nabla w d x \\
=\tau^{q-p} \mu\left(\int_{\Omega} a\left|v_{*}\right|^{q-2} v_{*} w d x+\int_{\partial \Omega} b\left|v_{*}\right|^{q-2} u_{*} w d \sigma\right)
\end{gathered}
$$

for all $w \in W^{1, p}(\Omega)$.

Finally, if we choose $\tau=(\lambda / \mu)^{1 /(q-p)}>0$, then $v_{*}=\tau u_{*}$ is an eigenfunction of problem (1.1) with eigenvalue $\lambda$. As has already been pointed out, $\lambda=0$ is an eigenvalue of problem (1.1). This conclude the proof.

\section{References}

[1] Adams, R.A., Fournier, J.J.F., Sobolev Spaces, second ed., Pure Appl. Math., 140, Academic Press, New York-London, 2003.

[2] Barbu, L., Moroşanu, G., Eigenvalues of the negative $(p, q)$ - Laplacian under a Steklovlike boundary condition, Complex Var. Elliptic Equ., 64(2019), no. 4, 685-700.

[3] Brezis, H., Functional Analysis, Sobolev Spaces and Partial Differential Equations, Springer, 2011.

[4] Casas, E., Fernández, L.A., A Green's formula for quasilinear elliptic operators, J. Math. Anal. Appl., 142(1989), 62-73.

[5] Fărcăşeanu, M., Eigenvalues for Finsler p-Laplacian with zero Dirichlet boundary condition, An. Sti. U. Ovid. Co.-Mat., 24(2016), no. 1, 231-242.

[6] Ferone, V., Kawohl, B., Remarks on Finsler-Laplacian, Proc. Am. Math. Soc., 137 (2008), no. 1, 247-253.

[7] Finsler, P., Über Kurven und Flachen in Allgemeinen Raumen, (Dissertation, Gottingen, 1918), Birkhäuser Verlag, Basel, 1951.

[8] Folland, G.B., Real Analysis: Modern Techniques and Their Applications (2nd ed.), Pure and Applied Mathematics, John Wiley \& Sons, Inc. New York, 1999.

[9] Giga, Y., Surface Evolution Equations. A Level Set Approach, Birkhäuser Verlag, Basel, 2006.

[10] Giusti, E., Direct Methods in the Calculus of Variations, 7, World Scientific, Singapore, 2003. 
[11] Lê, A., Eigenvalue problems for p-Laplacian, Nonlinear Anal. TMA., 64(2006), 10571099.

[12] Lindqvist P., Notes on p-Laplace equation, University of Jyväskylä, Lectures notes, 2006.

[13] Minkowski, H., Allgemeine Lehrsatze uber die konvexen Polyeder, Nachrichten von der Gesellschaft der Wissenschaften zu Göttingen, 1897, 198-219.

[14] Ôtani, M., Existence and nonexistence of nontrivial solutions of some nonlinear degenerate elliptic equations, J. Funct. Anal., 76(1988), 140-159.

[15] Taylor, J.E., Crystalline variational problems, Bulletin of the American Mathematical Society, 84(1978), 568-588.

[16] Zeidler, E., Nonlinear Functional Analysis and its Applications: II/B, Nonlinear Monotone Operators, Springer, New York, 1989.

Luminiţa Barbu

"Ovidius" University,

Faculty of Mathematics and Computer Sciences

124 Mamaia Blvd, 900527 Constanţa, Romania

e-mail: Ibarbu@univ-ovidius.ro 